\title{
PATIENT ASSESSMENT IN A\&E: AUDIT RESULTS
}

\section{Jacqueline Pennington, Staff Nurse; Barbara Teague, Clinical Audit Manager; WD Malone, Locum Consultant in A\&E Furness General Hospital}

\section{INTRODUCTION}

The term 'patient assessment' (PA) in Furness General Hospital (FGH) refers to medical, nursing and other activities surrounding patients who might or will be admitted to wards, but excluding those patients who self-present to A\&E. Patients for assessment may be for any of the inhouse specialities, and come from a variety of sources, principally GP referrals to duty inhouse teams but also from medical clinics, domiciliary visits, and other less frequently used routes. From the nursing point of view, all patients are cared for by A\&E nurses, and from a medical point of view patients are treated by doctors on that day's duty speciality team (unless the patient is extremely ill, in which case an A\&E doctor attends immediately). When patients arrive they are generally seen first by the houseman then by the $\mathrm{SHO}$, and in turn by the registrar. Besides this hierarchy of review, there are also reassessments undertaken after X-rays and ECGs (sometimes more than one reassessment after further x-rays and repeat ECGs).

Recently a separate system was set up by the orthopaedic consultants at FGH. The workload had increased because patients were also being received from Westmorland General Hospital (WGH). The system devised was that patients from WGH were transferred directly to the orthopaedic ward at FGH without stopping in A \& E for assessment. There have been no waits recorded in A\&E for these patients, and no problems have come to light in the system.

Changes within the trust occur frequently. Many of these changes have a direct effect on patient care (and waiting times in A\&E) which are not immediately obvious. For example, the SHO surgical rota was altered recently such that the SHO in surgery now frequently covers orthopaedics, surgery, theatre, wards and paediatric surgery at the same time. This widespread duty elsewhere makes the SHO less available for patient assessment in A\&E. Multi-disciplinary meetings over the last year had revealed that the PA workload had become sufficiently high at times as to draw all the nursing staff from $A \& E$ patient care, A\&E patients getting no nursing care at all, or short cuts being taken (sub-optimal care). Nursing staff experienced the conflict of undertaking patient assessment work at the expense of A\&E work, and found this to be a frustrating experience. This has affected morale. Another side effect of this was that the process of triage was frequently stopped, hence failing a national standard. Waiting times in A\&E have increased.

As the hospital approaches its capacity, and the medical unit becomes overwhelmed by the volume of work, operational systems, decisions and priorities are made unilaterally, A\&E losing control of its own workload. No single person appears to have a complete overview at any one time (the information for this does not appear to be available).

All problems are compounded during the months of August, September, February and March when the whole junior medical staff rotate. This four-month period is not a short episode. It is $33 \%$ of the year.

For these reasons, we decided to conduct a prospective audit of PA patients in several specialities. Details and information were collected sequentially by nursing staff.

\section{PURPOSE OF AUDIT}

Aim: to review current operational aspects of PA within A\&E

Objectives: identify existing guidelines for working practices relate current practice to guidelines

quantify and qualify any current inherent constraints within the PA system

use all data for discussion with appropriate directorates

\section{MATERIALS \& METHOD}

A data collection proforma was designed by one of the A\&E nurses. This was left on the nursing station inside the A\&E treatment area for completion each day. From 5 February to 31 March 2001, patient details and problems regarding times in $A \& E$ were recorded on the proforma by nursing staff.

Patients who spent more than six hours in A\&E were excluded from the audit as we understand that this data is collected on a routine basis by the trust as a national data requirement.

Independently from this study, Clinical Incident Report Forms were completed by individual A\&E staff members. Those completed and submitted from 1 February 2001 to June 2001 were reviewed. Those relating to patient assessment problems were identified and categorised. The complete reports are not included in this audit, but are available for review to interested parties.

\section{RESULTS}

\section{Guidelines}

No medical assessment guidelines, operational systems guidelines, or guidelines for any of the other specialties could 
be located within the A\&E department (save for orthopaedic patients from WGH direct to FGH ward).

\section{Audit Sample}

Information was recorded on the audit proforma in respect of 79 cases where a long wait had occurred.

The A\&E Admission Book was used by the clinical audit department to cross-reference patient details and timings. On perusal it was apparent that some of the patients who had experienced a long wait in $A \& E$ had not been included in the audit, possibly because of the busy workload and shortage of time. When these factors are taken into account, the audit only portrays a 'snapshot' of some of the waits and problems encountered during this period.

The 79 waits audited comprised 72 patients who were admitted to the wards via A\&E and 7 patients $(9 \%)$ who were discharged home (Figure 1). Seventy-seven percent were medical speciality patients (Figure 2 ).

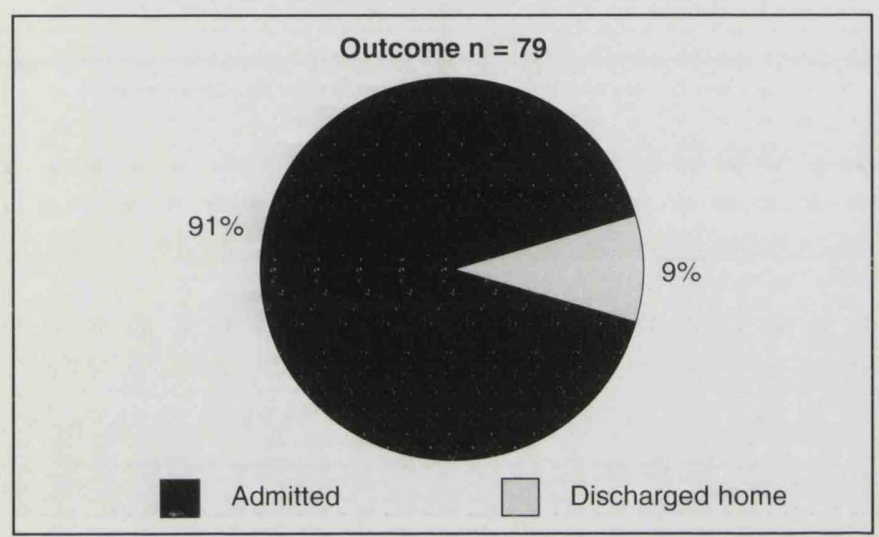

Figure 1

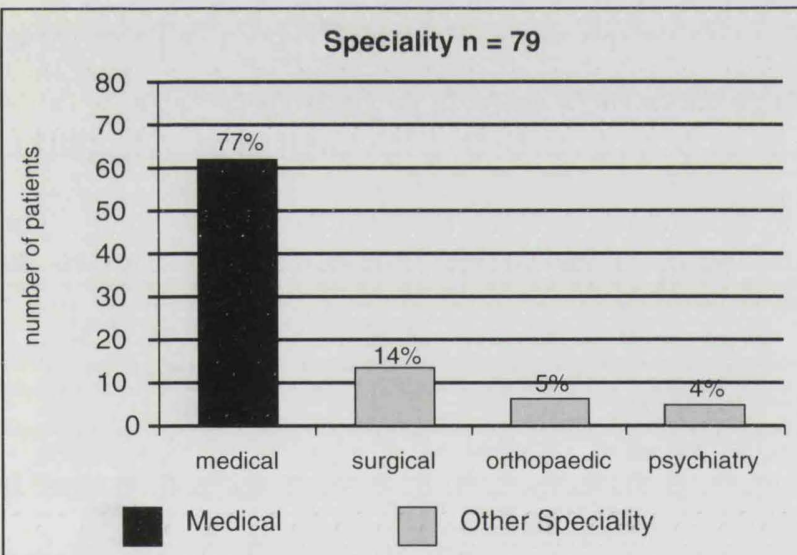

Figure 2

\section{Patient assessments for medical specialty}

Figure 3 highlights that $42(69 \%)$ of the 61 patients for medical assessment presented between 12 noon and $9 \mathrm{pm}$. Figure 4 illustrates that overall only four of the medical assessments $(7 \%)$ were discharged home.

Twenty-two $(39 \%)$ of the 57 medical assessment patients who were admitted had to wait in A\&E until a bed became available (ie bed shortage problem). This had an impact on the backlog of patients waiting to be seen. On many occasions during the audit period the bed shortage resulted in a queue of patients waiting to be admitted, a lack of trolleys in $\mathrm{A} \& \mathrm{E}$, complete blockage of the department and a shortage of space. Triage was frequently abandoned, and it was common for nurses to be unavailable to care for $\mathrm{A} \& \mathrm{E}$ patients.

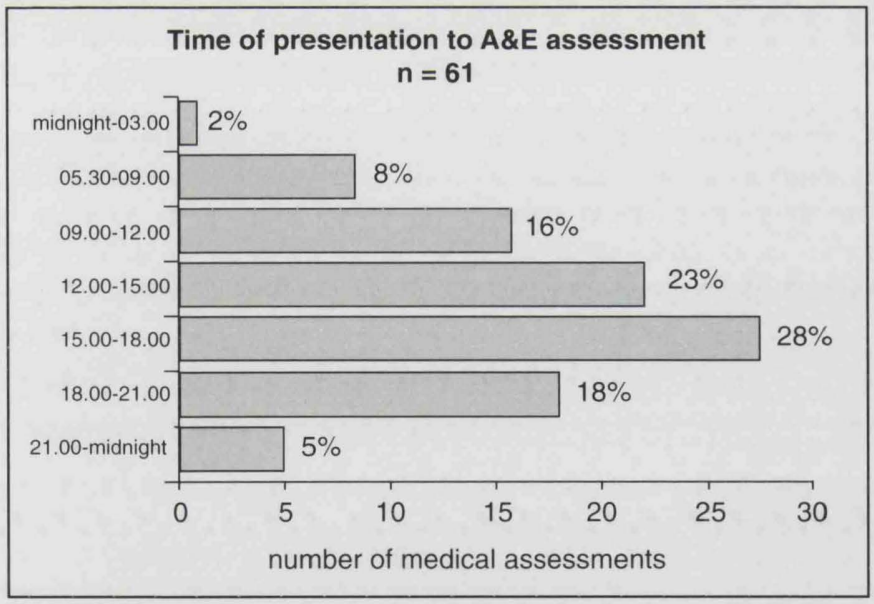

Figure 3

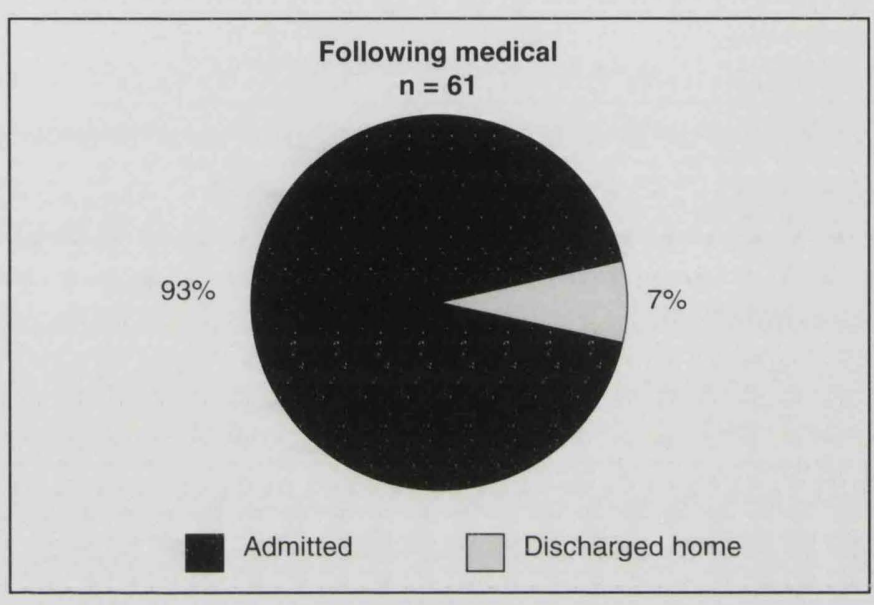

Figure 4

Thirty-five $(61 \%)$ medical assessment patients were held up in A\&E for reasons unrelated to bed shortages, namely:

patients for medical assessment arrive in groups $\quad=6$

the length of time taken to clerk/assess patient in $\mathrm{A} \& \mathrm{E}=3$

doctors could not attend A\&E because they were

on a ward round

$=9$

doctors were busy on the ward dealing with ill patients

$=8$

doctor left $\mathrm{A} \& \mathrm{E}$ in response to a 222 bleep for a cardiac arrest

doctor left patients waiting in A\&E to certify a death on the ward

doctor was called from A\&E by their consultant for rota discussion

tardy referral of a patient to the medical SHO

waiting for documentation of casenotes for a patient who was to be admitted

Length of time in A\&E for medical assessments requiring admission

As stated previously, patients who spent six hours or more in A\&E were excluded from the audit. Figure 5 shows the peak of waits due to bed shortage was 12 noon- $6 \mathrm{pm}$ while the spread of delays due to medical speciality being unavailable was wider (9am-9pm) and of greater number (Figure 6).

Figures 7 and 8 illustrate that more patients waited in A\&E (and for longer periods) because of the unavailability of speciality team doctors than for bed shortages. 


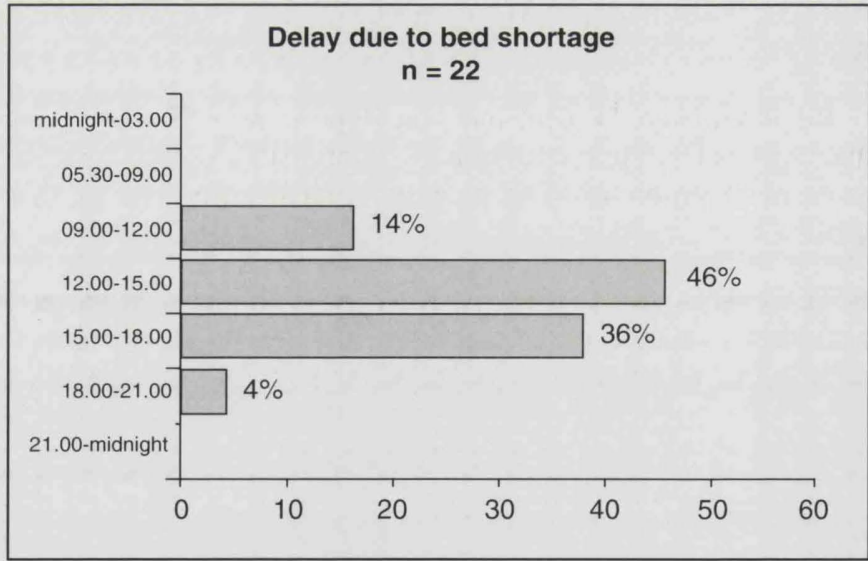

Figure 5

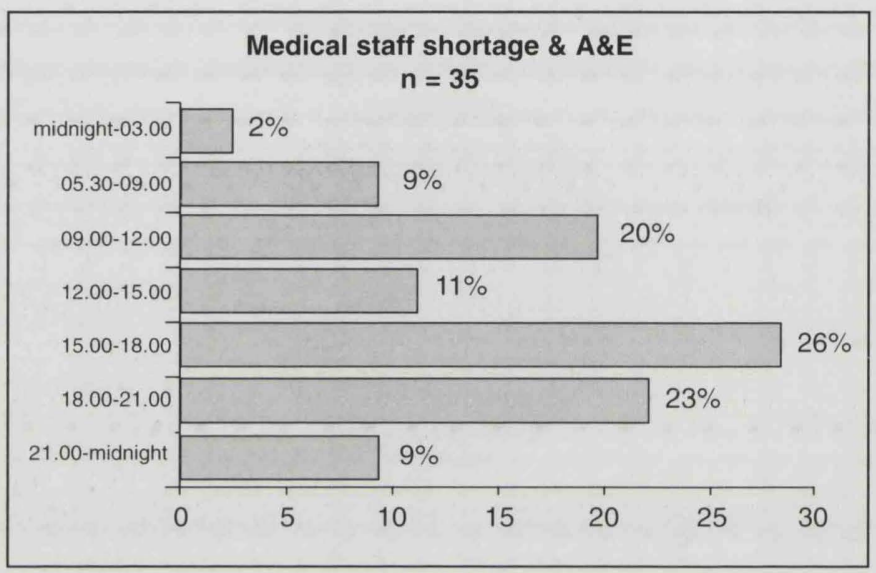

Figure 6

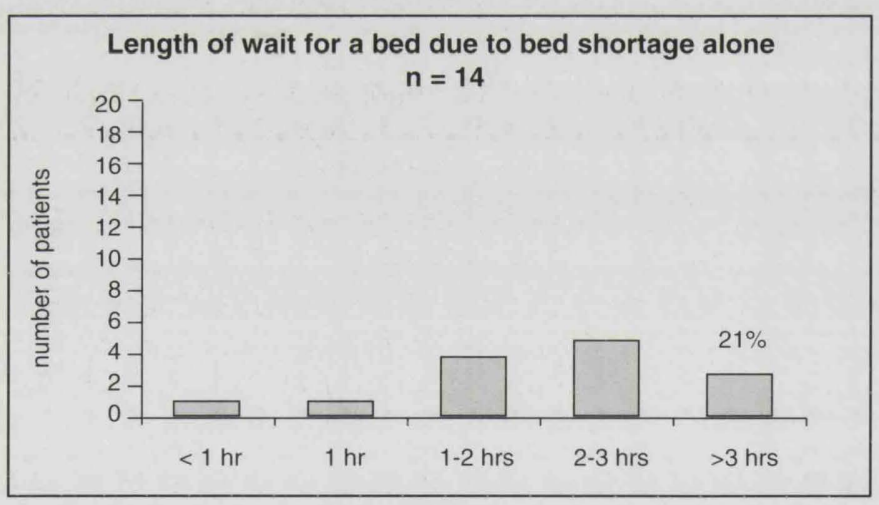

Figure 7

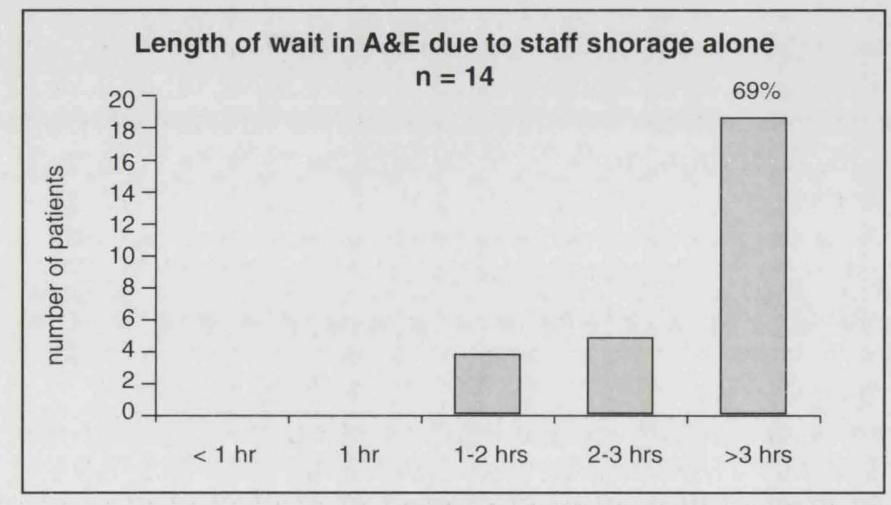

Figure 8
Specialty assessments in surgery, orthopaedics and psychiatry

These patients represent a smaller percentage of the total (23\% - Figure 2) but patient waits were of a similar pattern to medical assessment patients (ie waits due principally to unavailability of speciality medical staff [Figures 9 and 10]).

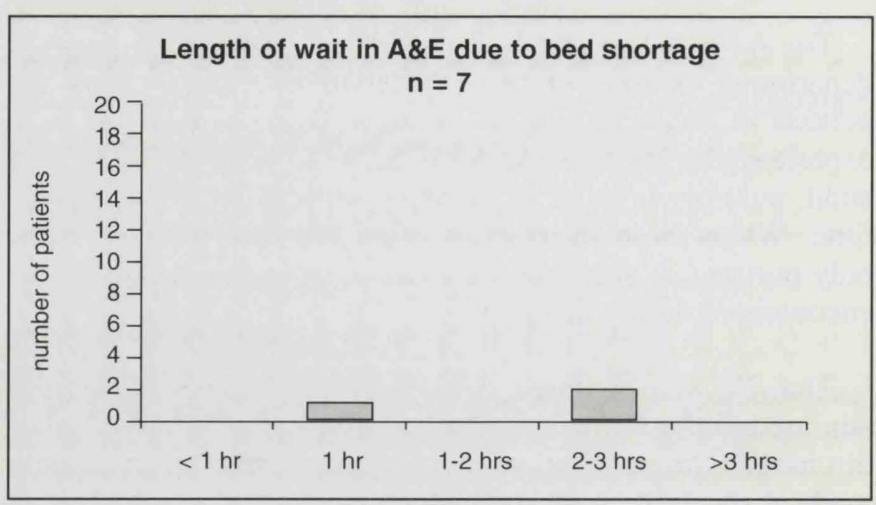

Figure 9

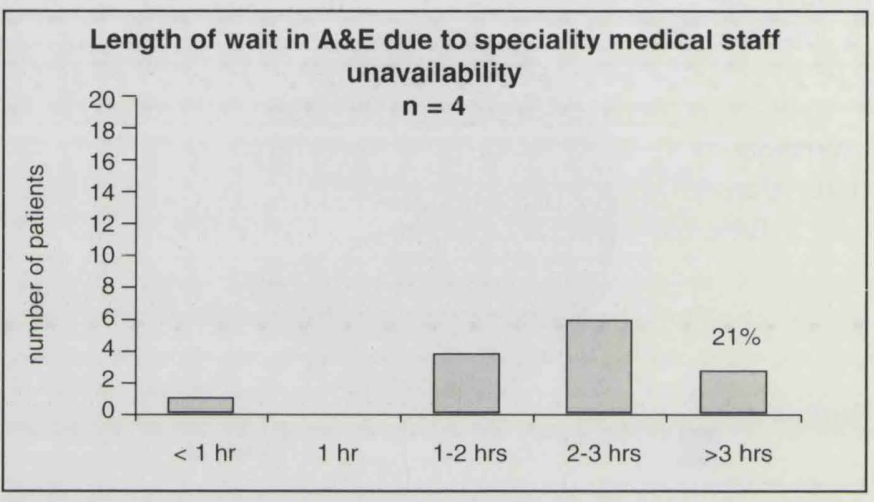

Figure 10

\section{REVIEW OF CLINICAL INCIDENT REPORTS RELATING TO PATIENT ASSESSMENT PROBLEMS (1 FEBRUARY 2001-8 JUNE 2001)}

The information supported the findings of the audit. The details are not included but are available for review to interested parties.

\section{DISCUSSION}

The audit highlighted an area of sub-optimal patient care. It has produced information which is new.

1 Waiting for specialty doctors to arrive was responsible for a greater percentage of patient waits than was bed shortage. Simply addressing the bed shortage situation will not have as great an effect on increasing the quality of patient care as addressing the problem of waits for speciality doctors.

This complex assessment system which caused significant patient delays led to the discharge of only a relatively small number of patients.

The literature indicates that A\&E SHOs can reliably assess patients requiring medical or surgical admission ${ }^{(1)}$. Further review by the medical or surgical $\mathrm{SHO}$ does not contribute to the management of patients.

The literature also indicates that inexperienced staff assessing patients results in inappropriate and insufficient care $^{(2)}$. It also supports a notion that those doctors making decisions about patient management should have no other 
commitments that prevent their immediate response to the A\&E department. There is further information indicating that the interface between $A \& E$ and acute general medicine should be addressed through the development of clear care pathways driven by explicit clinical standards.

The Faculty of A\&E issues guidance on coping with winter and emergency pressures and states very clearly that the pressures of bed shortages for the acute medical patients and their subsequent queuing within the hospital must not interfere with the optimal A\&E service, nor act as a constraint on any of the $A \& E$ services which run concurrently ${ }^{(3)}$.

2 The audit identified peak attendance times for patients. The logical steps would be to adjust medical speciality doctors' rotas to be available at those times. This may require significant organisational effort, but would certainly contribute to patient-centred care (a national quality issue).

3 The audit also demonstrated clearly that it has been false to regard the medical bed shortage as a single-phase issue. It is a complex issue, and there are many queues and much recirculation within those queues. Each of these queues will have its own limiting factors and control. Each needs to be considered in terms of the others. To alter one in isolation from the others could worsen the situation.

These complex pathways involve many healthcare workers, each of whom will be probably be able to offer significant information on the controlling factors. A 'bottom up' design should lead to the optimal solution. The hierarchy of operation and planning will need to go from this level through to trust board level via inter-directorate discussion. Multi-disciplinary involvement is the key to success.

Involving all staff out to the periphery of these care pathways will help address the problem of staff losing control of their own workload (and hence increased stress) and of the consequent decrease in morale.

It is recognised that the audit is presented from an A\&E point of view. The next most appropriate step would be to present the information to other directorates for their view and exchange of information.

4 It appears that the measurement of a single waiting time for an assumed single queue of patients awaiting speciality assessment in A\&E has seriously misled interpretation of the situation; solutions and resources may be correspondingly misplaced.

\section{CONCLUSIONS}

The audit has revealed that hospital bed shortage is not the most significant cause for patients waiting in $A \& E$ for specialty assessment. Delays in the majority of cases are due to the prioritisation of junior doctors' work of the speciality team to whom the patient is referred.

Medical assessment patients represent $77 \%$ of the total. The numbers of surgical, orthopaedic and psychiatric patients were fewer, but waits experienced by the specialities were also principally because the doctors were busy on the ward, or slow to answer their bleeps.

The arena of assessment of patients in A\&E has been shown to be a multiphase complex multi-factorial system. The care of A\&E patients is severely compromised by the current operational methods, and has led to widespread frustration and lowering of morale of $\mathrm{A} \& \mathrm{E}$ staff, and contributed to $\mathrm{A} \& \mathrm{E}$ failing to meet national triage criteria, and to longer waits for A\&E patients.

Review of the Clinical Incident Reports reveals significant numbers of reports relating appropriately to patient assessment problems. The data supports the findings of this audit.

There are no known operational or clinical guidelines/ pathways available (except for orthopaedic WGH transfers). Addressing these issues may well lead to improved patient care.

\section{RECOMMENDATIONS}

1) Optimal care for ALL patients to be a prime driving force

2) Current working practices need to be changed for optimal care for both A \& E patients and patients awaiting speciality assessment

3) Interdirectorate discussions should assess the current service and possible improvements such as:

- development of operational management systems and clinical care pathways

- how A\&E might regain control of its own workload

- decision-making team for medical assessments in A\&E throughout the peak patient attendance times (3pm-9pm)

- develop mechanisms to deal with complaints from patients and relatives when long queues develop

- review other appropriate pathways (avoiding A\&E) for patients awaiting assessment from outpatient medical clinics, domiciliary visits, or those with terminal illness

- expanding the bed management system in operation at night to the daytime, in which a nominated nursing sister finds beds for patients (and in so doing relieves congestion in A\&E). This role should cover finding beds for all specialties

- review the merits of the orthopaedic system of transfer from WGH to the FGH ward, bypassing assessment in A\&E. Consider application elsewhere

- giving authority to A\&E doctors to admit directly (for self-referred A\&E patients only)

- reviewing the possibility of moving patient assessment area from A\&E to a space currently occupied by day case (with its own entrance) and managed by the medical directorate.

3) Regular contact between all involved directorates, with regular feedback and audit to monitor practice.

\section{REFERENCES}

1) O'Connor PM et al. Unnecessary delays in accident and emergency department: do medical and surgical senior house officers need to vet admissions? J Acc \& Emerg Med 1995; $12: 251-254$

2) Coffey T, Mythen M (eds) NHS Front line - visions for 2010. Innovative approaches to integrating emergency care. London: The New Health Network

3) BAEM (P/1/01): http://www.baem.org.uk/winter.htm

\section{Acknowledgements.}

The authors wish to thank all the A\&E nursing staff for diligently completing the audit proformas, and Jackie Gray for her word processing skills in preparing the paper. 